Supporting Information

\title{
Realization of High Energy Density Sodium-Ion Hybrid Capacitors through Interface-Engineering of Pseudocapacitive 3D-CoO-NrGO Hybrid Anodes
}

\author{
Wenliang Feng a, b, Venkata Sai Avvaru ${ }^{\text {a, c }}$, Rudi Ruben Maça ${ }^{\text {a, c }}$, Steven J. Hinder ${ }^{\text {, }}$, Miguel \\ Castillo Rodríguez a, Vinodkumar Etacheri ${ }^{\text {a* }}$
}

${ }^{a}$ Electrochemistry Division, IMDEA Materials Institute, C/ Eric Kandel 2, Getafe, Madrid 28906, Spain.

${ }^{\text {b }}$ Departamento de Ciencia de Materiales, Universidad Politécnica de Madrid, E.T.S. de Ingenieros de Caminos, 28040 Madrid, Spain.

c Facultad de Ciencias, Universidad Autónoma de Madrid, C/ Francisco Tomás y Valiente, 7, 28049, Madrid, Spain.

d Surface Analysis Laboratory, Faculty of Engineering and Physical Sciences University of Surrey Guildford, Surrey GU2 7XH, United Kingdom (UK)

*E-mail: vinodkumar.etacheri@imdea.org 
Graphene oxide synthesis: Graphene oxide (GO) was synthesized through improved Hummers' method. Briefly, $1.0 \mathrm{~g}$ of graphite flakes (98\%, 50 mesh, Georg H. LUH Gmbh) and $6.0 \mathrm{~g}$ of $\mathrm{KMnO}_{4}(99 \%$, Sigma-Aldrich) were added successively into a 9:1 mixture of concentrated $\mathrm{H}_{2} \mathrm{SO}_{4} / \mathrm{H}_{3} \mathrm{PO}_{4}$ (98\%, Sigma-Aldrich). The reaction system was then heated to $50{ }^{\circ} \mathrm{C}$ under stirring for $12 \mathrm{~h}$. After cooling to room temperature, $200 \mathrm{~g}$ of ice was added into the reaction mixture followed by $2.0 \mathrm{~mL}$ of $30 \% \mathrm{H}_{2} \mathrm{O}_{2}$. The precipitate was separated by centrifugation (4000 rpm for $30 \mathrm{~min}$ ) washed in succession with $200 \mathrm{~mL}$ of 37\% $\mathrm{HCl}$ (98\%, Sigma-Aldrich), $200 \mathrm{~mL}$ of water and $200 \mathrm{~mL}$ of ethanol. The resulting material was finally dried in a vacuum oven at $80^{\circ} \mathrm{C}$ for $12 \mathrm{~h}$.

CoO loading calculation: TGA curve of 3D-CoO-NrGO under air flow shows a total weight loss of $30.9 \%$, taking $100 \mathrm{mg}$ of $3 \mathrm{D}-\mathrm{CoO}-\mathrm{NrGO}$ as an example, thus the residual of $69.1 \mathrm{mg}$ is $\mathrm{Co}_{3} \mathrm{O}_{4}$. Then, the weight of $\mathrm{CoO}$ loaded in the $3 \mathrm{D}-\mathrm{CoO}-\mathrm{NrGO}$ composites can be calculated through the following relationships:

$$
\begin{gathered}
\mathrm{Co}_{3} \mathrm{O}_{4} \leftrightarrow 3 \mathrm{CoO}+0.5 \mathrm{O}_{2} \\
\frac{W_{2}}{M_{2}}=\frac{W_{1}}{M_{1}} \times 3
\end{gathered}
$$

Where, $W_{1}$ is weight of residual, and $W_{2}$ is the weight of $\mathrm{CoO}$ in 3D-CoO-NrGO. $M_{1}$ and $M_{2}$ are the molar mass of $\mathrm{Co}_{3} \mathrm{O}_{4}$ and $\mathrm{CoO}$, respectively. 

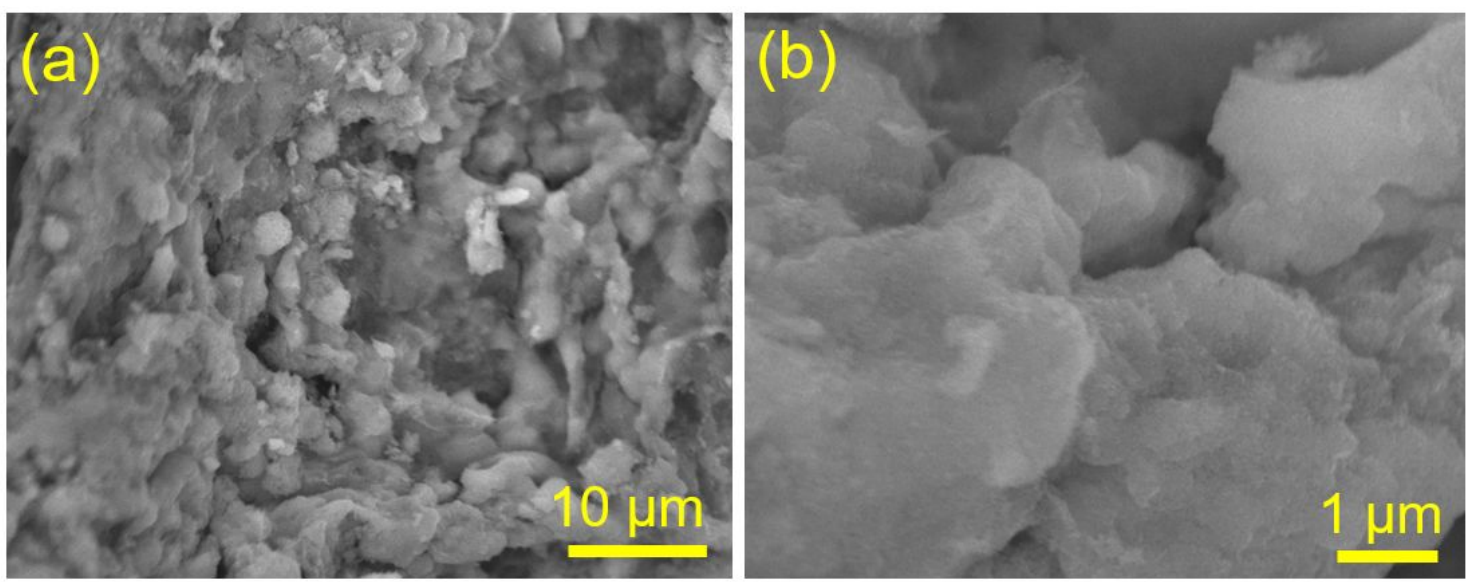

Figure S1. SEM images of 3D-(NH) $\mathrm{Co}_{8}\left(\mathrm{Co}_{3}\right)_{6}(\mathrm{OH})_{6} \cdot 4 \mathrm{H}_{2} \mathrm{O}-\mathrm{NrGO}$.

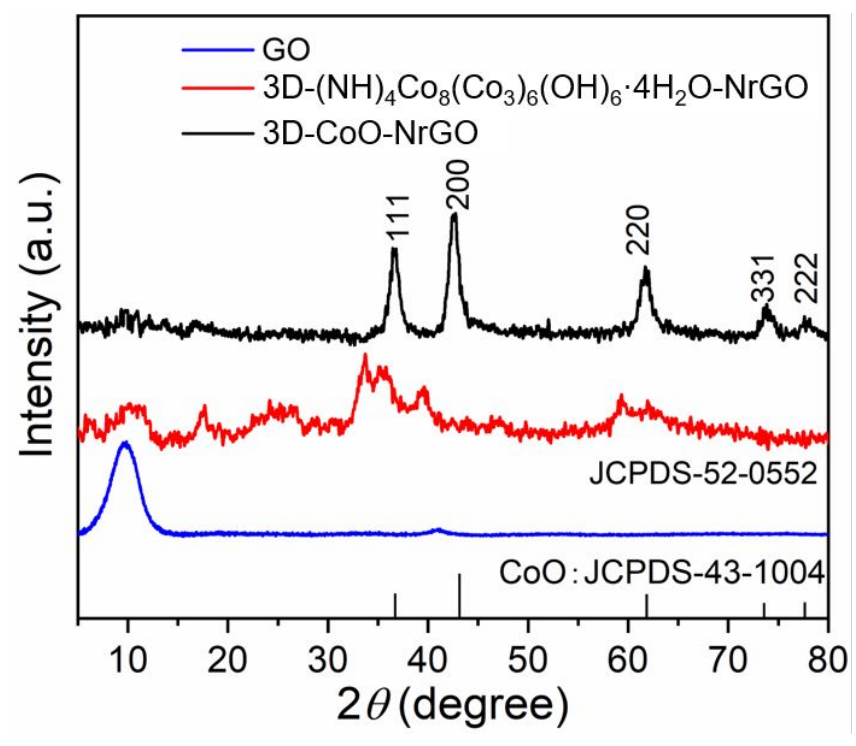

Figure S2. XRD patterns of GO, 3D-(NH) ${ }_{4} \mathrm{Co}_{8}\left(\mathrm{Co}_{3}\right)_{6}(\mathrm{OH})_{6} \cdot 4 \mathrm{H}_{2} \mathrm{O}-\mathrm{NrGO}$ and 3D-CoO-NrGO. 


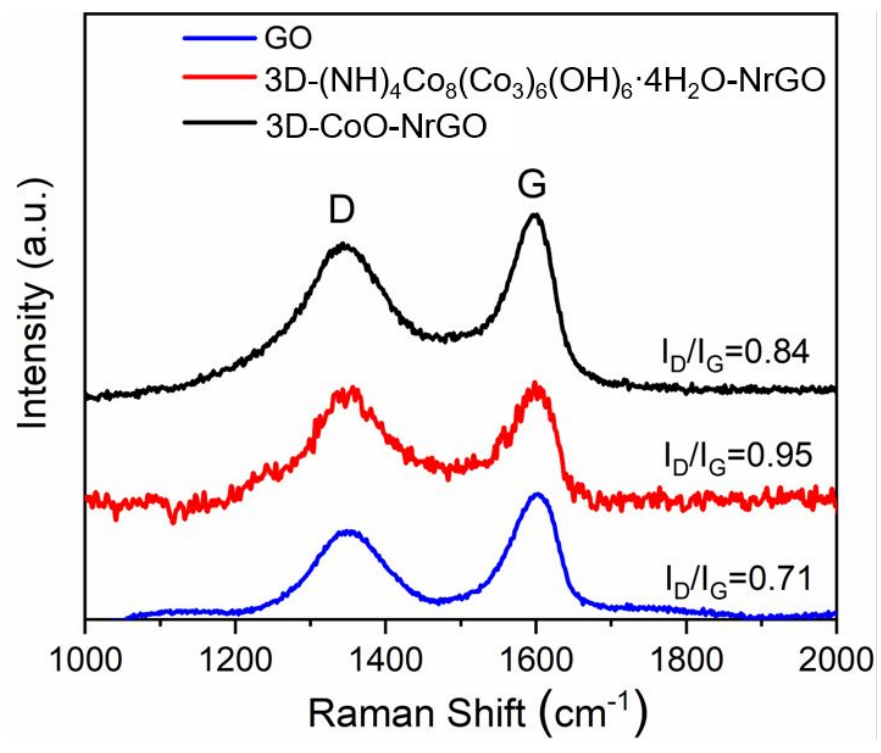

Figure S3. Raman spectra of GO, 3D-(NH) $)_{4} \mathrm{Co}_{8}\left(\mathrm{Co}_{3}\right)_{6}(\mathrm{OH})_{6} \cdot 4 \mathrm{H}_{2} \mathrm{O}-\mathrm{NrGO}$ and $3 \mathrm{D}-\mathrm{CoO}-\mathrm{NrGO}$.

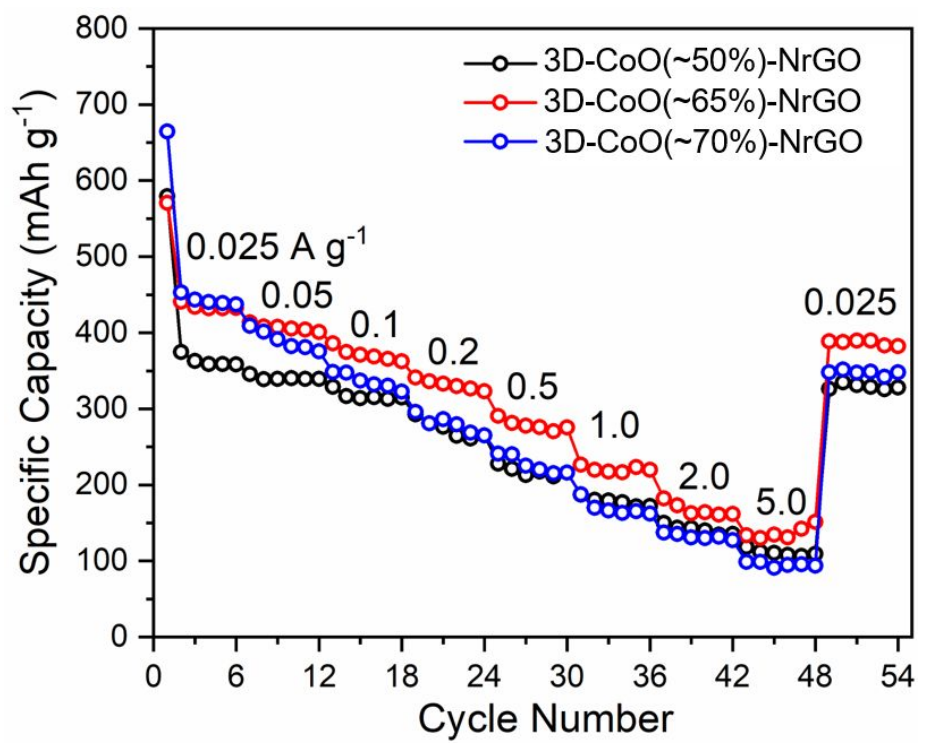

Figure S4. Galvanostatic rate performance of @3D-CoO-NrGO anodes containing different $\mathrm{CoO}$ loadings. 


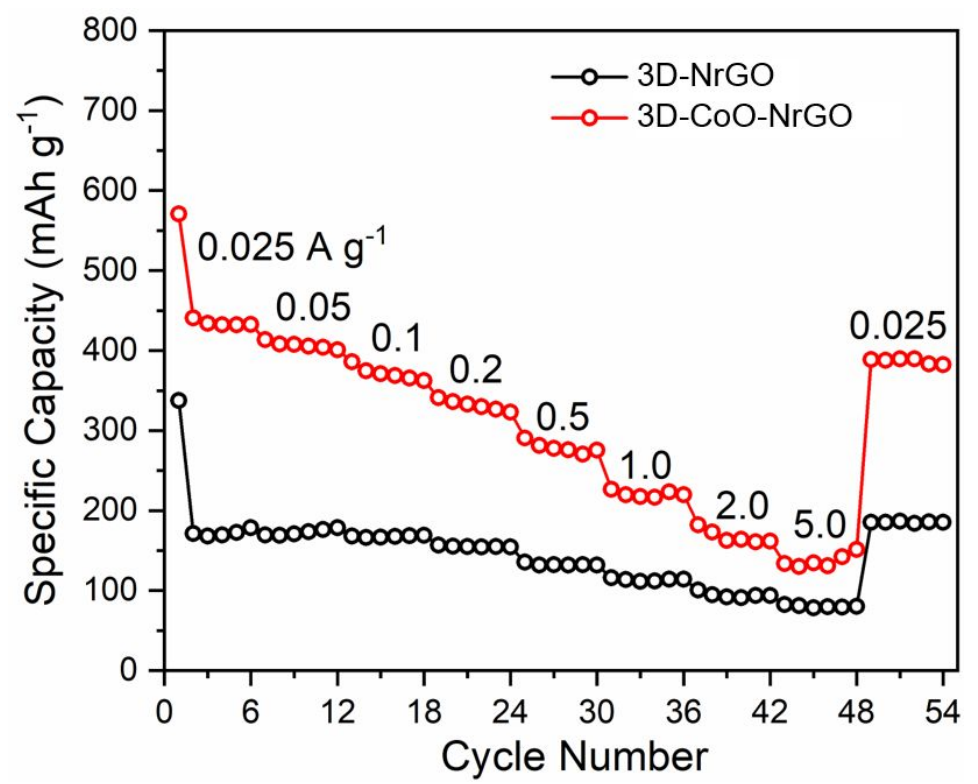

Figure S5. Galvanostatic rate performance of 3D-NrGO and 3D-CoO-NrGO anodes.
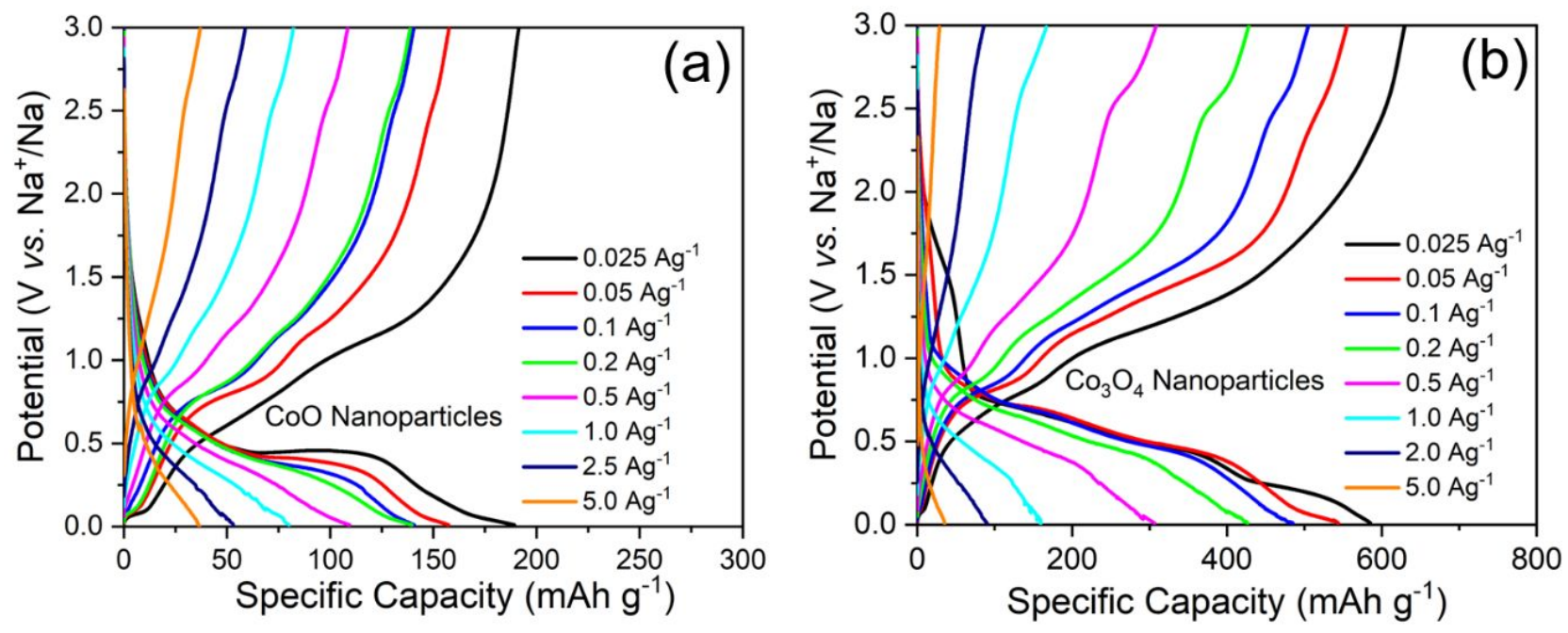

Figure S6. Voltage profiles of $\mathrm{CoO}$ and $\mathrm{Co}_{3} \mathrm{O}_{4}$ nanoparticle anodes at various current rates. 

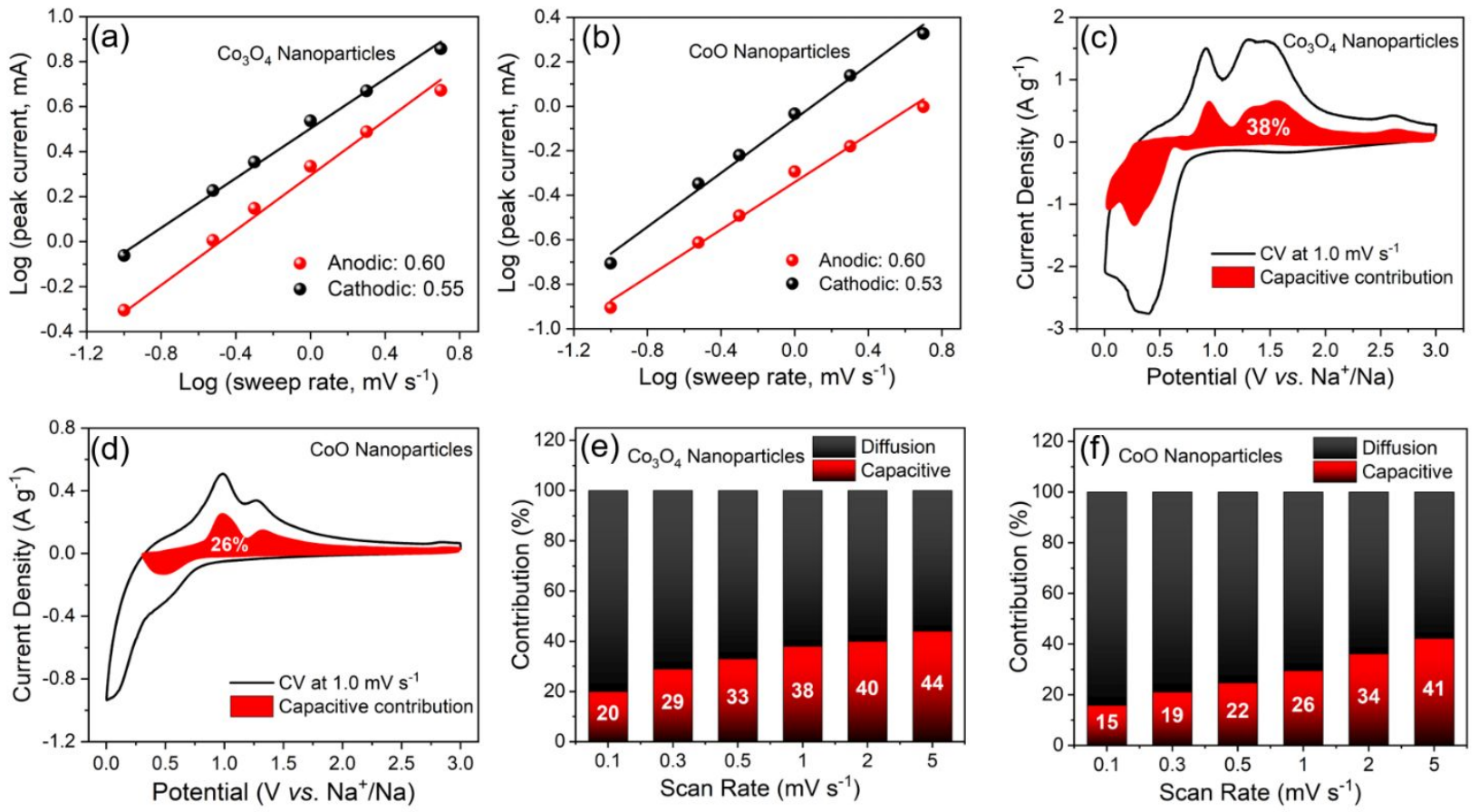

Figure S7. Peak current dependence of the scan rate of (a) $\mathrm{Co}_{3} \mathrm{O}_{4}$ and (b) $\mathrm{CoO}$ anodes.

Pseudocapacitive contribution at a scan rate of $1.0 \mathrm{mV} \cdot \mathrm{s}^{-1}$ of (c) $\mathrm{Co}_{3} \mathrm{O}_{4}$ and (d) $\mathrm{CoO}$ anodes.

Capacitive contribution percentages at various scan rates of (e) $\mathrm{Co}_{3} \mathrm{O}_{4}$ and (f) $\mathrm{CoO}$ anodes.

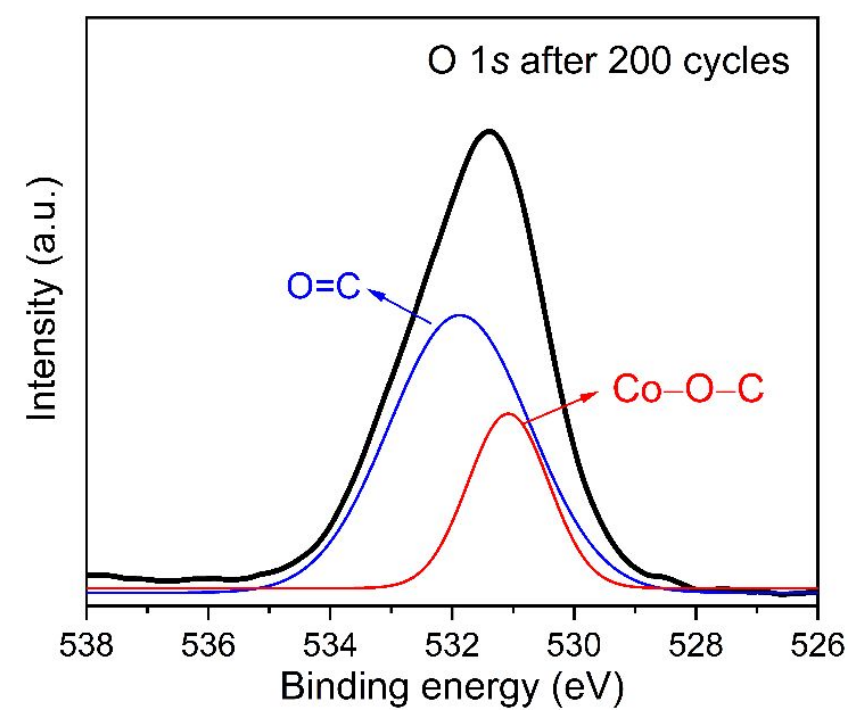

Figure S8. High-resolution O 1s spectra of 3D-CoO-NrGO electrode after 200 charge-discharge cycles. 


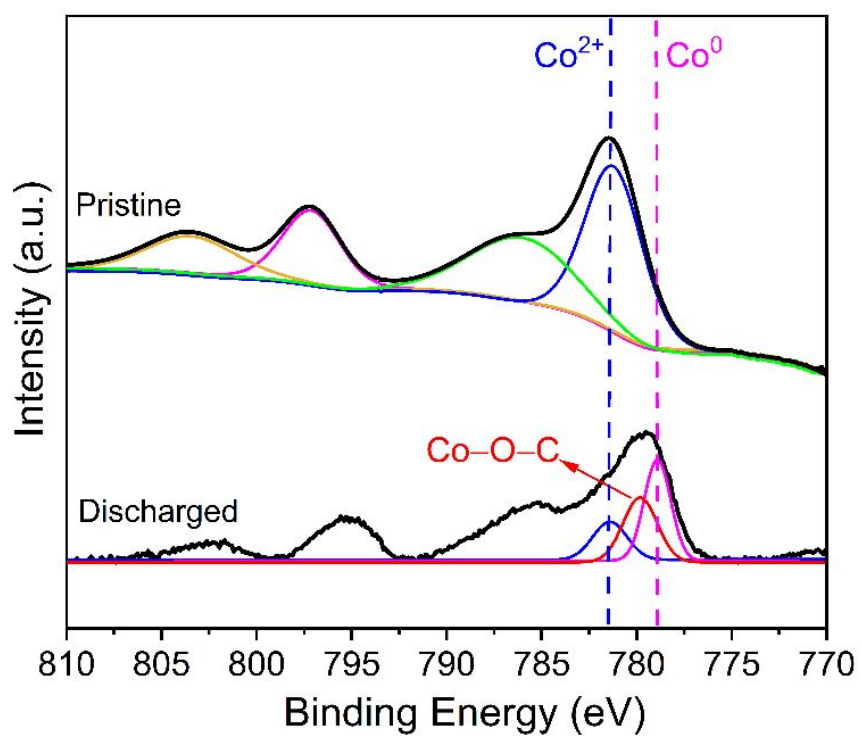

Figure S9. High-resolution Co 2p XPS spectra of pristine and fully discharged 3D-CoO-NrGO electrodes.

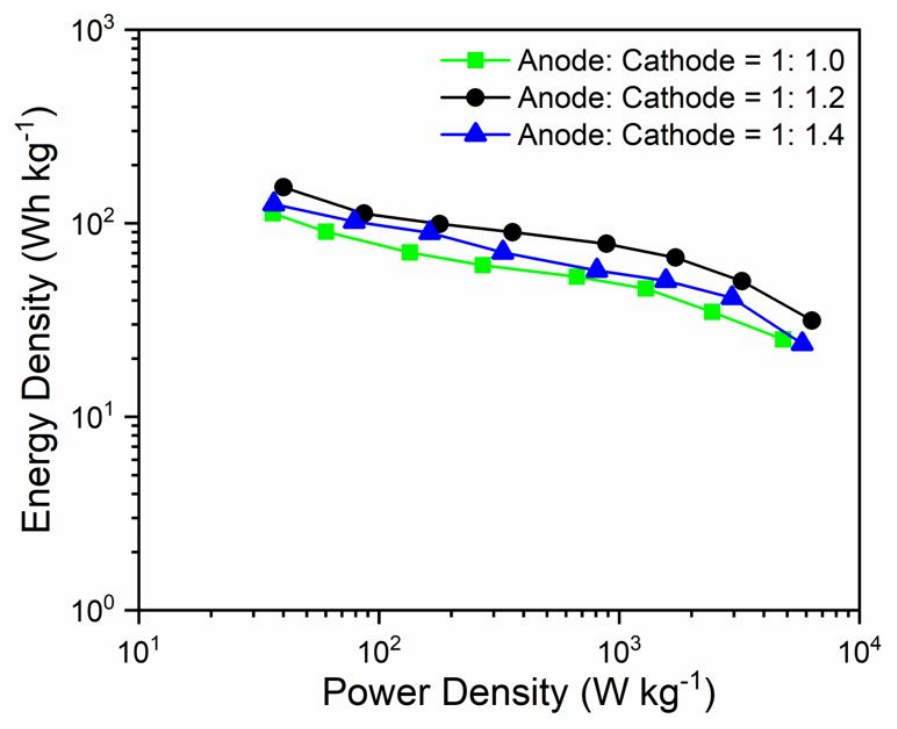

Figure S10. Ragone plot of 3D-CoO-NrGO//AC SHC based on different active mass ratio between 3D-CoO-NrGO anode and AC cathode. 


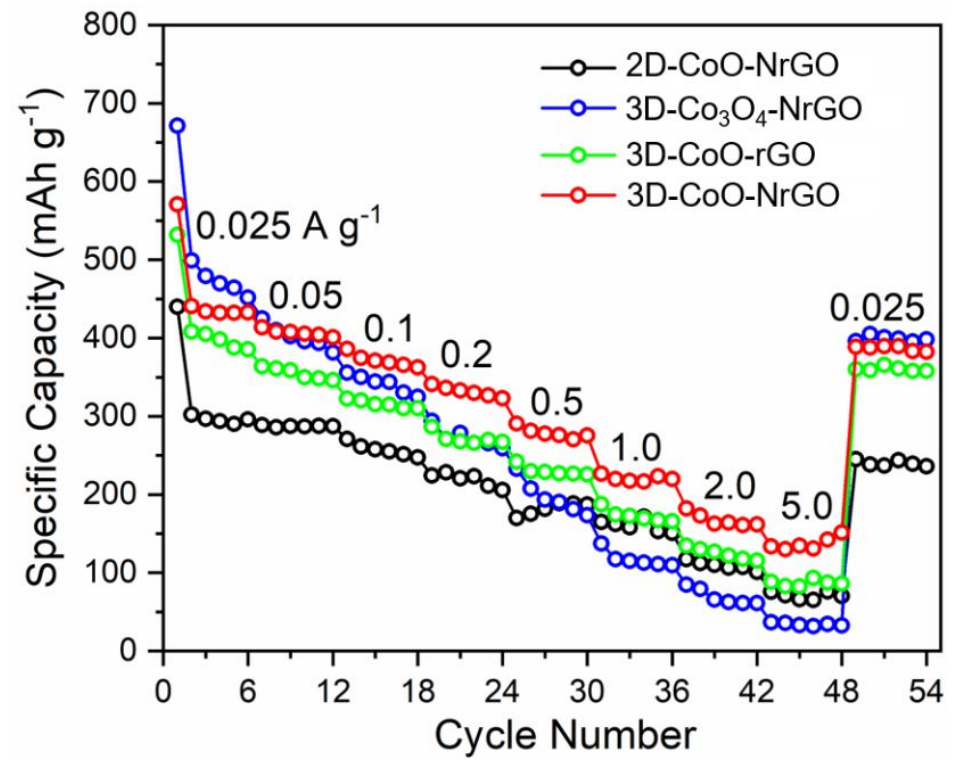

Figure S11. Galvanostatic rate performance of 2D-CoO-NrGO, 3D-Co $\mathrm{C}_{4}-\mathrm{NrGO}$

3D-CoO-rGO and 3D-CoO-NrGO anodes.

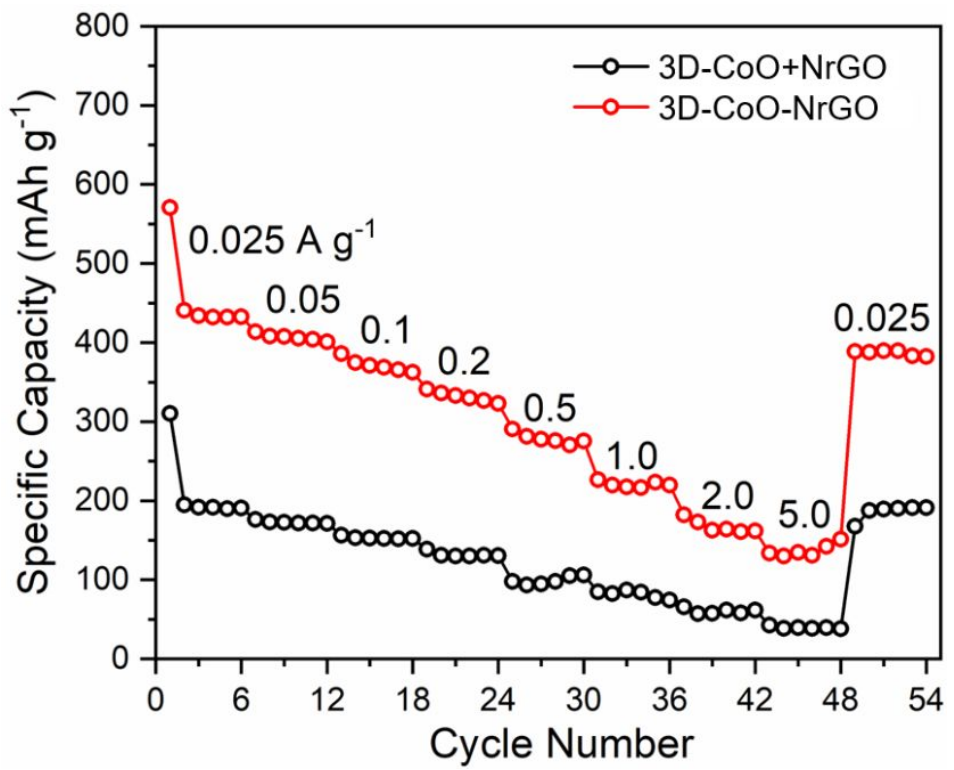

Figure S12. Galvanostatic rate performance of $3 \mathrm{D}-\mathrm{CoO}+\mathrm{NrGO}$ and $3 \mathrm{D}-\mathrm{CoO}-\mathrm{NrGO}$ anodes. 
Table S1. A comparison of the rate performance of the 3D-CoO-NrGO anode with previously reported Co based anodes for sodium ion batteries

\begin{tabular}{|c|c|c|c|c|c|}
\hline Anodes & $\begin{array}{c}\text { Voltage } \\
\text { (V) }\end{array}$ & $\begin{array}{c}\text { Specific capacity } \\
\left(\mathrm{mAh} \mathrm{g}^{-1}\right)\end{array}$ & $\begin{array}{l}\text { Current density } \\
\qquad\left(\mathrm{A} \cdot \mathrm{g}^{-1}\right)\end{array}$ & $\begin{array}{c}\text { Capacity } \\
\text { retention/ cycles }\end{array}$ & Ref \\
\hline $\mathrm{Co}_{3} \mathrm{O}_{4}$ Nanoplatelets & $0.01-3.0$ & $\begin{array}{l}415 \\
160\end{array}$ & $\begin{array}{c}0.089 \\
4.47\end{array}$ & $60 \% / 50$ & 1 \\
\hline $\mathrm{Co}_{3} \mathrm{O}_{4} / \mathrm{CNTs}$ & $0.01-3.0$ & $\begin{array}{l}410 \\
190\end{array}$ & $\begin{array}{c}0.05 \\
3.2\end{array}$ & $96 \% / 100$ & 2 \\
\hline $\mathrm{Co}_{3} \mathrm{O}_{4}$ fibers & $0.01-3.0$ & 578 & 0.09 & $68 \% / 30$ & 3 \\
\hline Pyramid-like $\mathrm{Co}_{3} \mathrm{O}_{4}$ & $0.01-3.0$ & 520 & 0.025 & $86 \% / 50$ & 4 \\
\hline $\begin{array}{c}\mathrm{Co}_{3} \mathrm{O}_{4} \text { nanocubes } / \\
\text { CNTs }\end{array}$ & $0.01-3.0$ & $\begin{array}{l}495 \\
258\end{array}$ & $\begin{array}{l}0.1 \\
1.6\end{array}$ & $76 \% / 100$ & 5 \\
\hline $\begin{array}{c}\mathrm{Co}_{3} \mathrm{O}_{4} \text { nanosheets/ } \\
\text { 3D graphene }\end{array}$ & $0.01-3.0$ & $\begin{array}{l}525 \\
82.3\end{array}$ & $\begin{array}{c}0.025 \\
0.5\end{array}$ & $92 \% / 50$ & 6 \\
\hline $\mathrm{Co}_{3} \mathrm{O}_{4} / \mathrm{NC}$ & $0.01-3.0$ & $\begin{array}{l}235 \\
130\end{array}$ & $\begin{array}{l}0.1 \\
4.0\end{array}$ & $91 \% / 100$ & 7 \\
\hline $\mathrm{CNF} / \mathrm{CoO}$ & $0.01-3.0$ & $\begin{array}{l}585 \\
423\end{array}$ & $\begin{array}{l}0.2 \\
1.0\end{array}$ & $95 \% / 100$ & 8 \\
\hline CoO microflowers & $0.01-3.0$ & $\begin{array}{l}340 \\
105\end{array}$ & $\begin{array}{l}0.091 \\
1.454\end{array}$ & $81 \% / 100$ & 9 \\
\hline $\mathrm{Co} / \mathrm{CoO} /$ Carbon & $0.01-3.0$ & $\begin{array}{l}307 \\
110\end{array}$ & $\begin{array}{l}0.1 \\
5.0\end{array}$ & $87 \% / 100$ & 10 \\
\hline $\mathrm{CoO} /$ Carbon black & $0.01-2.5$ & 300 & 0.089 & $60 \% / 50$ & 11 \\
\hline 3D-CoO-NrGO & $0.01-3.0$ & $\begin{array}{l}445 \\
135\end{array}$ & $\begin{array}{c}0.025 \\
5.0\end{array}$ & $93 \% / 200$ & $\begin{array}{l}\text { This } \\
\text { work }\end{array}$ \\
\hline
\end{tabular}


Table S2. Energy and power density comparison of 3D-CoO-NrGO//AC with the earlier reported SHC devices.

\begin{tabular}{|c|c|c|c|c|c|}
\hline SHC devices & $\begin{array}{c}\text { Voltage } \\
\text { (V) }\end{array}$ & $\begin{array}{l}\text { Energy density } \\
\quad\left(\mathrm{Wh} \mathrm{kg}^{-1}\right)\end{array}$ & $\begin{array}{l}\text { Power density } \\
\quad\left(\mathrm{Wh} \mathrm{kg}^{-1}\right)\end{array}$ & $\begin{array}{c}\text { Capacitance } \\
\text { retention/ cycles }\end{array}$ & Ref \\
\hline $\mathrm{TiO}_{2} / / \mathrm{AC}$ & $1.0-4.0$ & $\begin{array}{l}68 \\
23\end{array}$ & $\begin{array}{c}625 \\
7500\end{array}$ & $80 \% / 10000$ & 12 \\
\hline TiO2@CNT@C//AC & $1.0-4.0$ & $\begin{array}{c}81 \\
37.9\end{array}$ & $\begin{array}{c}126 \\
12400\end{array}$ & $83 \% / 5000$ & 13 \\
\hline MWTOG//AC & $1.0-3.8$ & $\begin{array}{l}64 \\
25\end{array}$ & $\begin{array}{c}56 \\
1357\end{array}$ & $90 \% / 10000$ & 14 \\
\hline $\mathrm{Na}_{2} \mathrm{Ti}_{2} \mathrm{O}_{5-\mathrm{x}} / / \mathrm{AC}$ & $1.0-3.8$ & $\begin{array}{l}70 \\
24\end{array}$ & $\begin{array}{c}240 \\
3600\end{array}$ & $82 \% / 5000$ & 15 \\
\hline $\mathrm{Nb}_{2} \mathrm{O}_{5} @ \mathrm{C} / \mathrm{rGO} / / \mathrm{AC}$ & $1.0-4.3$ & $\begin{array}{c}76 \\
6\end{array}$ & $\begin{array}{c}80 \\
20800\end{array}$ & $<70 \% / 2000$ & 16 \\
\hline MXenes//AC & $1.0-3.8$ & $\begin{array}{l}80 \\
42\end{array}$ & $\begin{array}{c}237 \\
6172\end{array}$ & $78 \% / 15000$ & 17 \\
\hline NHPC//NHPAC & $0-4.0$ & $\begin{array}{c}115 \\
20\end{array}$ & $\begin{array}{c}200 \\
4000\end{array}$ & $<50 \% / 10000$ & 18 \\
\hline 3D-CoO-NrGO//AC & $1.0-4.0$ & $\begin{array}{c}153 \\
31\end{array}$ & $\begin{array}{c}41 \\
6357\end{array}$ & $81 \% / 5000$ & $\begin{array}{l}\text { This } \\
\text { work }\end{array}$ \\
\hline
\end{tabular}

\section{References}

(1) Longoni, G.; Fiore, M.; Kim, J. H.; Jung, Y. H.; Kim, D. K.; Mari, C. M.; Ruffo, R. $\mathrm{Co}_{3} \mathrm{O}_{4}$ Negative Electrode Material for Rechargeable Sodium Ion Batteries: an Investigation of Conversion Reaction Mechanism and Morphology-Performances Correlations. J. Power Sources 2016, 332, 42-50.

(2) Rahman, M. M.; Sultana, I.; Chen, Z.; Srikanth, M.; Li, L. H.; Dai, X. J.; Chen, Y. Ex situ Electrochemical Sodiation/Desodiation Observation of $\mathrm{Co}_{3} \mathrm{O}_{4}$ Anchored Carbon Nanotubes: a High Performance Sodium-Ion Battery Anode Produced by Pulsed Plasma in a Liquid. Nanoscale 2015, 7, 13088-13095. 
(3) Santangelo, S.; Fiore, M.; Pantò, F.; Stelitano, S.; Marelli, M.; Frontera, P.; Antonucci, P.; Longoni, G.; Ruffo, R. Electro-Spun $\mathrm{Co}_{3} \mathrm{O}_{4}$ Anode Material for Na-Ion Rechargeable Batteries. Solid State Ionics 2017, 309, 41-47.

(4) Rahman, M. M.; Glushenkov, A. M.; Ramireddy, T.; Chen, Y. Electrochemical Investigation of Sodium Reactivity with Nanostructured $\mathrm{Co}_{3} \mathrm{O}_{4}$ for Sodium-Ion Batteries. Chem. Commun. 2014, 50, 5057-5060.

(5) Wang, Z.; Zhang, S.; Yue, L.; Wu, B.; Mi, J. Synthesis of $\mathrm{Co}_{3} \mathrm{O}_{4}$ Nanocubes/CNTs Composite with Enhanced Sodium Storage Performance. Solid State Ionics 2017, 312, $32-37$.

(6) Liu, Y. G.; Cheng, Z. Y.; Sun, H. Y.; Arandiyan, H.; Li, J. P.; Ahmad, M. Mesoporous $\mathrm{Co}_{3} \mathrm{O}_{4}$ Sheets/3D Graphene Networks Nanohybrids for High-Performance Sodium-Ion Battery Anode. J. Power Sources 2015, 273, 878-884.

(7) Xu, H.; Zhu, G.; Hao, B. Metal-Organic Frameworks Derived Flower-Like $\mathrm{Co}_{3} \mathrm{O}_{4} /$ Nitrogen Doped Graphite Carbon Hybrid for High-Performance Sodium-Ion Batteries. J. Mater. Sci. Technol. 2019, 35, 100-108.

(8) Jiang, J.; Ma, C.; Ma, T.; Zhu, J.; Liu, J.; Yang, G.; Yang, Y. A Novel CoO Hierarchical Morphologies on Carbon Nanofiber for Improved Reversibility as Binder-Free Anodes in Lithium/Sodium Ion Batteries. J. Alloy Compd. 2019, 794, 385-395.

(9) Chang, L.; Wang, K.; Huang, L. A.; He, Z.; Zhu, S.; Chen, M.; Shao, H.; Wang, J. Hierarchical $\mathrm{CoO}$ Microflower Film with Excellent Electrochemical Lithium/Sodium Storage Performance. J. Mater. Chem. A 2017, 5, 20892-20902.

(10) Kaneti, Y. V.; Zhang, J.; He, Y. B.; Wang, Z.; Tanaka, S.; Hossain, M. S.; Pan, Z. Z.; Xiang, B.; Yang, Q. H.; Yamauchi, Y. Fabrication of an MOF-Derived HeteroatomDoped $\mathrm{Co} / \mathrm{CoO} / \mathrm{Carbon}$ Hybrid with Superior Sodium Storage Performance for SodiumIon Batteries. J. Mater. Chem. A 2017, 5, 15356-15366.

(11) Yu, H.; Brown, Z. L.; Wei, C.; Obrovac, M. N. Thermoelectrochemical Activation of $\mathrm{CoO}$ in Na Cells. J. Electrochem. Soc. 2018, 165, 1595-1600.

(12) Babu, B.; Ullattil, S. G.; Prasannachandran, R.; Kavil, J.; Periyat, P.; Shaijumon, M. M. $\mathrm{Ti}^{3+}$ Induced Brown $\mathrm{TiO}_{2}$ Nanotubes for High Performance Sodium-Ion Hybrid Capacitors. ACS Sustain. Chem. Eng. 2018, 6, 5401-5412. 
(13) Zhu, Y. E.; Yang, L.; Sheng, J.; Chen, Y.; Gu, H.; Wei, J.; Zhou, Z. Fast Sodium Storage in $\mathrm{TiO}_{2} @ \mathrm{CNT} @ \mathrm{C}$ Nanorods for High-Performance Na-Ion Capacitors. Adv. Energy Mater. 2017, 7, No. 1701222.

(14) Le, Z.; Liu, F.; Nie, P.; Li, X.; Liu, X.; Bian, Z.; Chen, G.; Wu, H. B.; Lu, Y. Pseudocapacitive Sodium Storage in Mesoporous Single-Crystal-Like $\mathrm{TiO}_{2}$-Graphene Nanocomposite Enables High-Performance Sodium-Ion Capacitors. ACS Nano 2017, 11, 2952-2960.

(15) Que, L. F.; Yu, F. D.; He, K. W.; Wang, Z. B.; Gu, D. M. Robust and Conductive $\mathrm{Na}_{2} \mathrm{Ti}_{2} \mathrm{O}_{5-\mathrm{x}}$ Nanowire Arrays for High-Performance Flexible Sodium-Ion Capacitor. Chem. Mater. 2017, 29, 9133-9141.

(16) Lim, E.; Jo, C.; Kim, M. S.; Kim, M. -H.; Chun, J.; Kim, H.; Park, J.; Roh, K. C.; Kang, K.; Yoon, S.; Lee, J. High-Performance Sodium-Ion Hybrid Supercapacitor Based on $\mathrm{Nb}_{2} \mathrm{O}_{5} @$ Carbon Core-Shell Nanoparticles and Reduced Graphene Oxide. Adv. Funct. Mater. 2016, 26, 3711-3719.

(17) Kurra, N.; Alhabeb, M.; Maleski, K.; Wang, C. H.; Alshareef, H. N.; Gogotsi, Y. BiStacked Titanium Carbide (MXene) Anodes for Hybrid Sodium-Ion Capacitors. ACS Energy Lett. 2018, 3, 2094-2100.

(18) Zou, K.; Cai, P.; Liu, C.; Li, J.; Gao, X.; Xu, L.; Zou, G.; Hou, H.; Liu, Z.; Ji, X. Kinetic Well-Matched Full-Carbon Sodium-Ion Capacitor. J. Mater. Chem. 2019, 7, 1354013549 . 